\title{
Self Protect: Um jogo para auxílio no ensino de conceitos relacionados a Segurança na Internet para Crianças e Adolescentes
}

\author{
Fernando Lucas de Oliveira Farias ${ }^{1}$, Neide Aparecida Alves de Medeiros ${ }^{1}$, Stênio Lúcio da \\ Rocha $^{1}$, Daniel Fonseca de Medeiros ${ }^{1}$, Edith Cristina da Nóbrega ${ }^{1}$, Aquiles Medeiros \\ Filgueira Burlamaqui ${ }^{2}$,Charles Andryê Galvão Madeira ${ }^{1}$
}

\author{
Instituto Metrópole Digital - Universidade Federal do Rio Grande do Norte (UFRN) ${ }^{1}$
}

Complexo Tecnológico de Engenharia - Universidade Federal do Rio Grande do Norte (UFRN) ${ }^{2}$

\{fernandoo.mcp, neidemedeiros, steniolucio1305, geodanielprof1, aquilesburlamaqui

\}@gmail.com, edithecn@ufrn.edu.br, charles@imd.ufrn.br

\begin{abstract}
The threats that children and adolescents use through the Internet use insecurely have gained notoriety and room for debate throughout the country. In this context, this paper aims to create a 2D platform game entitled Self Protect to assist in the teaching of concepts related to internet security for children and adolescents. The development of the game followed the defined methodological line of elementary tetrad (aesthetics, narrative, mechanics and technology). This experience resulted in a greater contact with the final audience and their observations were relevant to the improvement of this playful solution, allowing us to reflect on how users absorb the lessons covered in the game.

Resumo. As ameaças originadas por meio do uso da internet de modo inseguro por crianças e adolescentes tem ganhado notoriedade e espaço em debates em todo país. Nesse contexto, este trabalho objetiva por meio da criação de um jogo de plataforma $2 D$ intitulado Self Protect auxiliar no ensino de conceitos relacionados a segurança na internet para crianças e adolescentes. O desenvolvimento do jogo seguiu a linha metodológica definida de tétrade elementar (estética, narrativa, mecânica e tecnologia). Esta experiência nos resultou um contato maior com o público final e suas observações foram relevantes para o aprimoramento desta solução lúdica permitindo refletir como os usuários absorvem os ensinamentos abordados no jogo.
\end{abstract}

\section{Introdução}

A segurança na internet é um tema transversal que precisa de maior atenção por parte da comunidade escolar, principalmente pela democratização do acesso à internet que chega a oito em cada dez crianças e adolescentes (82\% - 24,3 milhões no Brasil) com idades entre 9 e 17 anos, bem como a proliferação de ameaças cibernéticas nocivas ao uso seguro da internet [Carvalho et al. 2017], [CGI.br 2017].

Segundo Ponte (2012), a forma com que crianças e adolescentes lidam com os diferentes tipos de riscos, e o que eles representam em termos de oportunidades, se altera de acordo com suas habilidades digitais, com a posição que o usuário assume na cadeia de comunicação e, ainda, varia de acordo com o contexto e o estilo de mediação exercido pelas famílias, professores e outros responsáveis. 
A abordagem de conceitos relacionados a segurança na internet: golpes e ataques na internet, códigos maliciosos (malware) e spam; podem ser classificados como temas transversais porque permeiam os diversos campos do conhecimento, contemplando questões sociais cotidianas, abordadas tangencialmente no jogo de plataforma 2D desenvolvido em consonância a temas transversais eleitos pelos Parâmetros Curriculares Nacionais (PCNs) [Silva et al. 2015], [Brasil 1998].

O jogo proposto foi desenvolvido utilizando a ferramenta Construct 2 desenvolvida pela Scirra, selecionada por não requerer conhecimento específico de uma linguagem de programação, ideal para trabalho em equipes multidisciplinares como a envolvida no projeto, bem como a exportação dos jogos em HyperText Markup Language (HTML 5), facilidade de aprendizado e a utilização do conceito WYSIWYG (What You See Is What You Get - o que você vê é o que você tem) [Silva 2017], [Andrade et al. 2012],[Medeiros; Silva; Aranha 2013].

O trabalho está organizado da seguinte forma: na Seção 2 são apresentados trabalhos relacionados ao uso de jogos digitais na educação; a Seção 3 apresenta a metodologia utilizada no desenvolvimento do jogo; a Seção 4 detalha os resultados obtidos após as crianças e adolescentes jogarem e o engajamento deles, por fim, a Seção 5 detalha as Conclusões e apresenta possibilidades para Trabalhos Futuros.

\section{Trabalhos relacionados}

A Aprendizagem baseada em jogos digitais surge no contexto educacional, como uma tendência pedagógica que vem transformando o desenho dos processos de ensino e aprendizagem na perspectiva da aprendizagem com jogos.

Segundo Tarouco et al. (2004), os jogos tem capacidade singular de divertir enquanto motivam, bem como são capazes de facilitar o aprendizado, ampliando a taxa de retenção do que foi ensinado no exercício das funções mentais e cognitivas do jogador, atuando como ferramentas instrucionais eficientes.

Santos et al. (2014), destaca a necessidade de conscientização, através de conteúdos educacionais, em relação aos riscos existentes na Internet, apresentando um Objeto de Aprendizagem sobre medidas de prevenção na Internet denominado "Prevenção na Internet" que apresenta conceitos sobre malware, spam, invasões e o assunto Engenharia Social, sendo este último desconhecido por grande parte dos alunos participantes do experimento. Os resultados indicam maior preocupação e motivação com a temática por parte dos aprendizes, bem como mudanças positivas em relação à atenção e conscientização sobre os riscos no uso da Internet.

A utilização do jogo Self Protect para auxílio no ensino de conceitos relacionados à segurança na internet para crianças e adolescentes, diferencia-se dos trabalhos relacionados pela abordagem tangencial, lúdica e interativa proporcionada pelo jogo digital, bem como pela carência de trabalhos que apresentem objetos de aprendizagem capazes de conscientizar sobre os riscos associados a códigos maliciosos e os benefícios da utilização adequada das ferramentas de segurança online.

\section{Metodologia}

O desenvolvimento do jogo Self Protect seguiu a linha metodológica proposta por Schell (2011) no que diz respeito à definição da tétrade elementar (Estética, narrativa, mecânica e tecnologia) do 
VIII Congresso Brasileiro de Informática na Educação (CBIE 2019)

Anais do XXV Workshop de Informática na Escola (WIE 2019)

jogo e Novak (2011) quanto às etapas para sua concepção (Conceito, pré-produção, protótipo, produção e pós-produção), conforme descrito na Tabela 1.

Tabela 1: Etapas da concepção do Jogo Self Protect

\begin{tabular}{|c|l|}
\hline Etapa & \multicolumn{1}{|c|}{ Descrição } \\
\hline Conceito & $\begin{array}{l}\text { A equipe multidisciplinar alocada no projeto elaborou um mapa mental }{ }^{1} \text {, a fim de estabelecer } \\
\text { conceitos relacionados a segurança na internet serem priorizados na proposta. Estes conceitos } \\
\text { foram extraídos em sua maioria de conteúdo instrucional }{ }^{2} \text { disponibilizado pelo NIC.br. }\end{array}$ \\
\hline Pré-produção & $\begin{array}{l}\text { O Short Game Design Document (SGDD) }{ }^{3} \text { ou documento de design do jogo foi elaborado para } \\
\text { facilitar o planejamento e a documentação mais robusta da história, estética, mecânicas, artes, } \\
\text { programação, músicas e sons presentes no jogo. }\end{array}$ \\
\hline Protótipo & $\begin{array}{l}\text { O protótipo foi desenvolvido utilizando a ferramenta Construct 2, estruturado inicialmente em } \\
\text { menu principal, fase 1 - ativando o antivírus, fase 2 - combatendo ameaças e fase 3 - vencendo o } \\
\text { chefão, permitindo que o aprendiz comprenda conceitos relacionados a worms, spyware, } \\
\text { keylogger, firewall, antivírus de forma lúdica e interativa. }\end{array}$ \\
\hline Produção & $\begin{array}{l}\text { O desenvolvimento do jogo foi realizado em três fases: Alpha (motor e interfaces completos, } \\
\text { permitindo que seja jogado do início ao fim), Beta (correção de bugs identificados na fase anterior, } \\
\text { fruto de testes realizados por membros do projeto e usuários participantes dos testes) e Ouro } \\
\text { (ajustes finais e publicação na Web). }\end{array}$ \\
\hline Pós-Produção & $\begin{array}{l}\text { A previsão é que sejam disponibilizadas novas versões do jogo com novas fases incluindo } \\
\text { Protegendo Contas e Senhas, Privacidade e Segurança em dispositivos móveis. }\end{array}$ \\
\hline
\end{tabular}

Fonte: Elaborado pelos autores

Figura 1: (a) Visão geral da Fase 1; (b) Visão geral da Fase 2; (c) Visão Geral da Fase 3

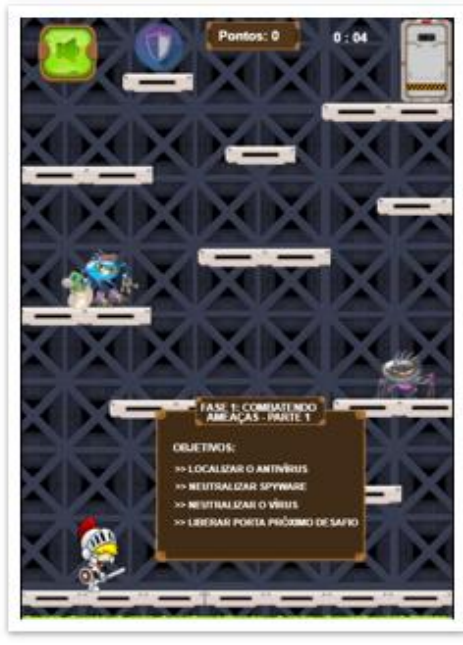

(a)

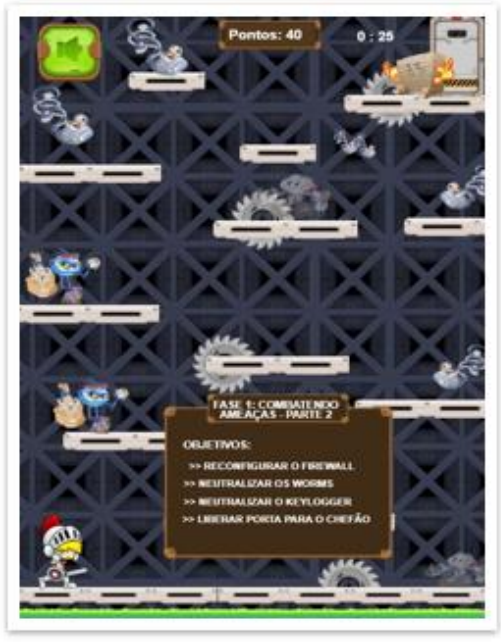

(b)

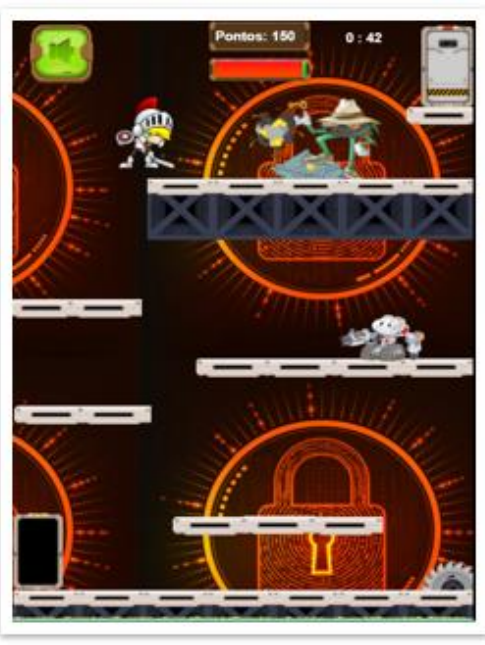

(c)

Fonte: Elaborado pelos autores

\footnotetext{
${ }^{1}$ Disponível em: < http://bit.ly/2zGToS8> Acesso em: 16 jul. 2018

2 Disponível em: $<$ https://cartilha.cert.br/> Acesso em: 16 jul. 2018

3 Disponível em: <http://bit.ly/2N3WQIp > Acesso em: 16 jul. 2018
} 
VIII Congresso Brasileiro de Informática na Educação (CBIE 2019)

Anais do XXV Workshop de Informática na Escola (WIE 2019)

\subsection{Estética}

A jogabilidade do Self Protect ${ }^{4}$ é baseada em um sistema de Jumping Game com cenários em Plataforma no qual o jogador deverá controlar um personagem utilizando teclas direcionais do teclado e/ou interagindo com touch de seu dispositivo móvel, esquivando-se das mais diversas ameaças: worms, keyloggers, spywares, ransomwares e buscando itens de segurança a exemplo de antivírus e firewall para neutralizar as ameaças existentes em cada fase em tempo pré-definidos.

O jogo é ambientado em fases (ver Figura 1a, Figura 1b e Figura 1c) utilizando sprites e layouts de uso comum, obtidos através do portal Game Art 2D $\mathrm{D}^{5}$, assim como imagens contidas na

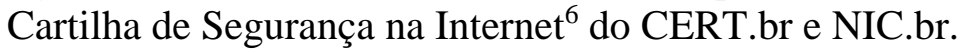

Ao final de cada fase, por cada ameaça neutralizada o jogador é pontuado contabilizando uma pontuação global que integrará um ranking a ser disponibilizado na versão 2.0 do protótipo. Este, listará a pontuação obtida pelo jogador e o tempo consumido na solução dos desafios dispostos nas fases do jogo.

Sempre que o jogador é abatido por uma ameaça presente na fase ativa ele é levado automaticamente para tela de Game over que contabiliza as ameaças neutralizadas e pontuação obtida até o momento, ilustrada na Figura 1a.

\subsection{Narrativa}

Um sistema de computadores foi infectado por uma série de malwares no momento em que seus programas de segurança encontravam-se inoperantes. Neste instante o antivírus encontrava-se desatualizado, o firewall desabilitado e o Backup do sistema inativo, ocasionando inúmeros transtornos e comportamentos inesperados ao sistema.

A infestação teve início ao acessar um website sem adoção de práticas seguras no uso da internet, resultando na infecção do sistema pelas ameaças constantes na Tabela 2. Cabendo agora ao protagonista atualizar e restabelecer o sistema, eliminando os malwares que estão comprometendo o pleno funcionamento.

Tabela 2: Malwares ilustrados no Jogo Self Protect

\begin{tabular}{|c|c|c|l|}
\hline Malware & \multicolumn{1}{|c|}{ Descrição $^{7}$} & Malware & \multicolumn{1}{c|}{ Descrição } \\
\hline $\begin{array}{l}\text { Programa de computador, } \\
\text { normalmente malicioso, } \\
\text { que se propaga inserindo } \\
\text { cópias de si mesmo e se } \\
\text { tornando parte de outros } \\
\text { programas e arquivos. }\end{array}$ & $\begin{array}{l}\text { Programa capaz de se } \\
\text { propagar automaticamente } \\
\text { pelas redes, enviando cópias } \\
\text { de si mesmo de computador } \\
\text { para computador. }\end{array}$ \\
\hline
\end{tabular}

\footnotetext{
${ }^{4}$ Disponível em: $<$ http://bit.ly/2NG3m9x $>$ Acesso em: 16 jul. 2018

${ }^{5}$ Disponível em: $<$ http://bit.ly/2mlom8X> Acesso em: 16 jul. 2018

${ }^{6}$ Disponível em: $<$ http://bit.ly/2NnFTZH $>$ Acesso em: 17 jul. 2018

${ }^{7}$ Disponível em: $<$ http://bit.ly/2NnFTZH $>$ Acesso em: 17 jul. 2018
} 
VIII Congresso Brasileiro de Informática na Educação (CBIE 2019)

Anais do XXV Workshop de Informática na Escola (WIE 2019)

\begin{tabular}{|c|l|l|l|}
\hline Malware & \multicolumn{1}{|c|}{ Descrição $^{7}$} & Malware & \multicolumn{1}{c|}{ Descrição } \\
\hline Spyware & $\begin{array}{l}\text { Programa projetado para } \\
\text { monitorar as atividades de } \\
\text { um sistema e enviar as } \\
\text { informações coletadas para } \\
\text { terceiros. }\end{array}$ & $\begin{array}{l}\text { Tipo de código malicioso que } \\
\text { torna inacessíveis os dados } \\
\text { armazenados em um } \\
\text { equipamento, geralmente } \\
\text { usando criptografia, e que } \\
\text { exige pagamento de resgate } \\
\text { (ransom) para restabelecer o } \\
\text { acesso ao usuário. }\end{array}$ \\
\hline
\end{tabular}

Fonte: Elaborado pelos autores

\subsection{Mecânicas}

Para o jogo foi escolhido mecânicas voltadas ao jogo de plataforma side-scrolling, com Jumping, recuperação de objetos chaves (antivírus e firewall), colisão com objetos (plataformas físicas e elementos de penalização), habilidade (pulo com controle e desvio de objetos), Puzzle - recolher objetos orientados nas fases para obter acesso à fase seguinte.

Tabela 3: Ferramentas segurança ilustradas no Jogo Self Protect

\begin{tabular}{|c|l|l|l|}
\hline $\begin{array}{c}\text { Ferramenta } \\
\text { Segurança }\end{array}$ & \multicolumn{1}{|c|}{$\begin{array}{c}\text { Fescrição } \\
\text { Segurança }\end{array}$} & Descrição \\
\hline Antivírus & $\begin{array}{l}\text { Tipo de ferramenta } \\
\text { antimalware desenvolvido } \\
\text { para detectar, anular e } \\
\text { eliminar de um computador } \\
\text { vírus outros tipos de códigos } \\
\text { maliciosos. }\end{array}$ & $\begin{array}{l}\text { Dispositivo de segurança } \\
\text { usado para dividir e } \\
\text { controlar o acesso entre } \\
\text { redes de computadores. }\end{array}$ \\
\hline
\end{tabular}

Fonte: Elaborado pelos autores

Ao iniciar o jogo utilizando o menu interativo, ver Figura 1c, o jogador deverá ativar o antivírus do sistema que foi desabilitado pelo malwares presentes na Fase 1, neste sentido, ele deverá, utilizando as habilidades de seu personagem, saltar utilizando as setas direcionais do teclado para subir os blocos dispostos na fase, desviando-se das ameaças que tentarão impedi-lo de alcançar o antivírus que está no topo do cenário.

Neste estágio, o personagem ainda não desenvolveu o poder de atacar com sua espada, bem como não poderá colidir com nenhuma ameaça, sob pena de morrer imediatamente após qualquer contato indo para tela de Game over, ver Figura 1a. Seu desafio consiste em até sessenta segundos ativar o antivírus do sistema, sem colidir com o vírus ou spyware que são as ameaças presentes nesta etapa que tentarão impedi-lo de coletar o item, logrando êxito será desbloqueada a porta para Fase 2, ver Figura 1b; onde o jogador enfrentará novos desafios para habilitar o Firewall do sistema que está comprometido.

\footnotetext{
8 Disponível em: <http://bit.ly/2zKFPkD> Acesso em: 17 jul. 2018

${ }^{9}$ Antimalware é uma ferramenta que procura detectar e, então, anular ou remover os códigos maliciosos de um computador.
} 
Ao iniciar a Fase 2, o jogador é exposto a novos níveis de dificuldade, que exigem paciência e resiliência para vencer todos os desafios propostos e alcançar o item de recompensa localizado na parte superior da fase, responsável por habilitar o Firewall do sistema, ver Figuras 1d e 4. Os principais desafios são desviar das serras que possuem velocidades variáveis, sendo que o personagem não poderá colidir em nenhum momento com esta ameaça, deverá coletar os antivírus existentes distribuídos no cenário que permitem neutralizar os Worms e Keyloggers que tentarão impedi-lo de concluir sua missão; coletados os antivírus desviando-se das ameaças, o jogador deverá alcançar o Firewall presente na porta superior da Fase 2 que será desbloqueada, dando acesso a última fase, onde enfrentará o chefão denominado Ransoware, que sequestrou os dados e backup do sistema.

Na última fase, o personagem desenvolve a habilidade de manipular o poder de sua espada que é acionado clicando-se com o mouse sob o alvo onde pretende lançar seu poder, ver Figura 5, sendo este fundamental para derrotar o chefão que impedirá o personagem de avançar e resgatar os dados sequestrados.

O principal desafio deste estágio é que o jogador precisará desferir diversos golpes com sua espada para derrotar o Ransoware que tentará a todo momento atingir o jogador com uma bola de fogo lançada por ele em intervalos aleatórios. À medida que o jogador acerta seu oponente uma barra de vida é exibida indicando o avanço na eliminação da ameaça. Após superar o chefão da Fase 3, o jogador encerra com sucesso a sua jornada que teve início com a ativação do antivirus na Fase 1, a habilitação do Firewall na Fase 2 e recuperação dos dados e do backup do sistema na última fase.

\subsection{Aspectos Pedagógicos}

A utilização de jogos digitais como artefatos pedagógicos lúdicos e motivadores, tem se destacado principalmente por sua capacidade de tornar o aprendiz protagonista nos processos de ensino e aprendizagem mediante sua postura como agente ativo na construção do seu conhecimento fruto de investigação e descoberta [Felicia 2012],[Falcão; Barbosa 2015].

Segundo Bruner (1960), a aprendizagem por descoberta consiste na possibilidade do estudante construir seu conhecimento mediante sua própria iniciativa, guiando-se por uma investigação autônoma disciplinada por artefatos, sendo que os conceitos não são transmitidos explicitamente pelo professor; ela é baseada no construtivismo que segundo Phillips (1972) permite ao sujeito desempenhar um papel ativo na construção do seu conhecimento e próprio desenvolvimento, fruto da interação entre o sujeito e os objetos/situações.

A proposta desenvolvida levou em consideração algumas habilidades relacionadas ao ensino de Computação para as etapas da Educação Básica propostas pela Sociedade Brasileira de Computação - SBC através do documento ${ }^{10}$ que trata dos referenciais de formação em computação para a Educação Básica, dentre elas podemos destacar no eixo Mundo Digital (MD) "Compreender princípios de segurança em computadores para evitar um uso inadequado, compreender as limitações e propiciar um uso mais seguro da internet em seus processos de pesquisa, uso de redes sociais e demais utilidades de seu cotidiano" [SBC 2017].

\footnotetext{
${ }^{10}$ Disponível em: <http://bit.ly/2LMcevX> Acesso em: 18 jul. 2018
} 
O Self Protect além de simular, divertir e advertir o usuário sobre alguns riscos contidos na internet, desperta o movimento sobre certas ações virtuais que podem ser refletidas no mundo real, colaborando para uma cultura de conscientização quanto a proteção dos dados pessoais.

\section{Resultados}

Após a disponibilização do protótipo do jogo desenvolvido, a avaliação da experiência do usuário foi realizada com crianças e adolescentes, através de um survey estruturado nas seguintes dimensões: Perfil do Jogador, Design, Jogabilidade, Mecânicas, Roteiro e Feedback do Jogador; baseado nas propostas de Krause et al. (2017) e Dias et al. (2016) utilizando a escala de Likert para questões relacionadas à percepção profícua dos jogadores acerca do Self Protect [Likert 1932 apud Silva, Melo e Tedesco 2016].

Gráfico 1: (a) Faixa Etária; (b) Escolaridade; (c) Instituição de Ensino dos Jogadores

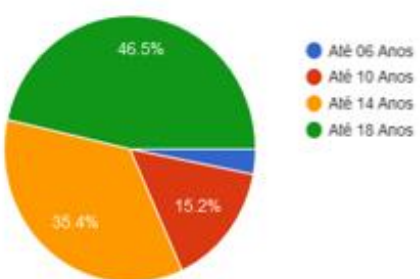

(a)

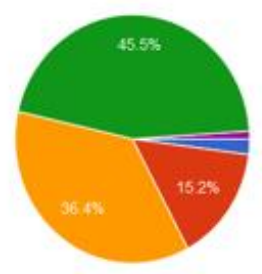

(b)

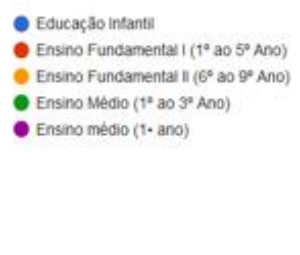

Tonte: Elaborado pelos autores

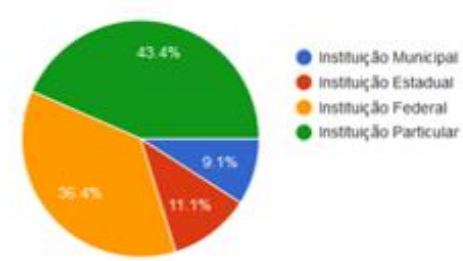

(c)

O jogo foi disponibilizado online para crianças e adolescentes, contabilizando 99 jogadores participantes do experimento; matriculados em instituições de ensino particular, municipal, estadual e federal com escolaridade no Ensino Fundamental I ( $1^{\circ}$ ao $5^{\circ}$ Ano), Fundamental II ( $6^{\circ}$ ao $9^{\circ}$ Ano) e Ensino Médio ( $1^{\circ}$ ao $3^{\circ}$ Ano), conforme dados "perfil do jogador" nos Gráfico 1a, Gráfico 1b e Gráfico 1c.

Na dimensão "Jogabilidade" foram avaliados aspectos relacionados ao êxito do jogador em terminar o jogo, nível de diversão (i), interesse em jogar novamente (ii), número de ameaças (iii) e dificuldade em eliminar as ameaças do jogo (iv), neste aspecto identificamos uma avaliação melhor das crianças em relação à percepção dos elementos de "Jogabilidade" reportada pelos adolescentes, como demonstram os Gráfico 2a e Gráfico 2b

Gráfico 2: (a) "Jogabilidade” pelas Crianças; (b) "Jogabilidade" pelos Adolescentes
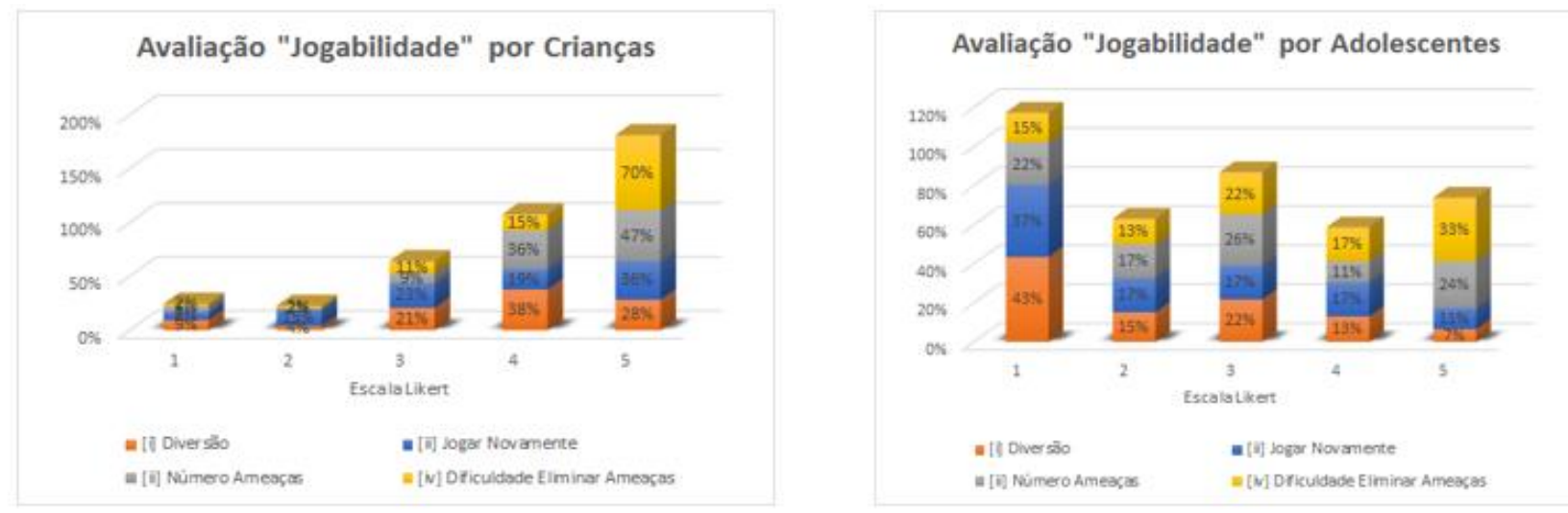

Fonte: Elaborado pelos autores 
A dimensão "Jogabilidade" foi indicada pelos usuários como aquela que demanda aprimoramentos por parte do time multidisciplinar responsável pelo desenvolvimento e manutenção evolutiva do projeto, principalmente relacionados à melhorias em algumas mecânicas do jogo que incluem: (a) ajustes no nível de dificuldade da fase 1 para fase 2, (b) facilidades para movimentação do personagem, (c) tutoriais que descrevam claramente os comandos para movimentação do personagem, (d) possibilidade de ataque com a espada desde início da aventura e (e) criação de uma barra de vida para o personagem.

Foram avaliados os aspectos positivos e negativos que os usuários gostariam de destacar no jogo educacional "Self Protect". As principais contribuições foram consolidadas na Tabela 4.

Tabela 4: Self Protect - Principais Aspectos Positivos e Negativos

\begin{tabular}{|c|c|c|c|}
\hline Aspecto & Contribuição & Aspecto & Contribuição \\
\hline \multirow{4}{*}{$\begin{array}{l}\text { Aspectos } \\
\text { Positivos }\end{array}$} & $\begin{array}{l}\text { "Jogabilidade boa, bastante intuitivo e } \\
\text { divertido" }\end{array}$ & \multirow{4}{*}{$\begin{array}{l}\text { Aspectos } \\
\text { Negativos }\end{array}$} & $\begin{array}{l}\text { "A dificuldade para conduzir o } \\
\text { bonequinho" }\end{array}$ \\
\hline & $\begin{array}{l}\text { "A quantidade de informação sobre } \\
\text { segurança fornecida ao jogador" }\end{array}$ & & $\begin{array}{l}\text { "Não consegui terminar, então achei muito } \\
\text { difícil" }\end{array}$ \\
\hline & $\begin{array}{l}\text { "Ajuda o aluno a aprender e visualizar } \\
\text { melhor os conceitos de segurança da } \\
\text { informação" }\end{array}$ & & $\begin{array}{l}\text { "Algumas fases possuem muitos inimigos, } \\
\text { o que dificulta um pouco para passar para } \\
\text { a próxima, principalmente se for uma } \\
\text { criança um pouco mais nova." }\end{array}$ \\
\hline & $\begin{array}{l}\text { "Dificuldade, perfeito para desafiar } \\
\text { amigos" }\end{array}$ & & $\begin{array}{l}\text { "O jogador morre mesmo sem chegar } \\
\text { perto das ameaças!" }\end{array}$ \\
\hline
\end{tabular}

Fonte: Elaborado pelos autores

Os aspectos tanto positivos quanto negativos foram contribuições valiosas enviadas pelos usuários que testaram o jogo desenvolvido em sua versão 1.0; sendo que estas foram analisadas, classificadas e incluídas em sua maioria no backlog $^{11}$ de novas funcionalidades e aprimoramentos para a versão 2.0 do Self Protect.

\section{Considerações finais}

No processo de concepção do jogo, nas etapas de conceito e pré-produção listadas na Tabela 1; destaque-se a produtividade e integração proporcionadas pela construção colaborativa tanto do mapa mental quanto do short game design document (SGDD) pela equipe do projeto.

Tais documentos, foram relevantes na definição das características do jogo e gerenciamento interativo de tais requisitos por parte da equipe multidisciplinar alocada no projeto; a construção de tais documentos consistiu numa etapa fundamental, atuando como norteadores nas etapas de iniciação, planejamento, monitoramento e controle e encerramento no Life Cycle Canvas (LLC) do projeto [Veras 2016]; bem como estruturação da narrativa/história, estética, mecânicas, artes/design, programação e sonoplastia presentes no software educacional desenvolvido.

\footnotetext{
11 Backlog pode ser classificado como uma lista de todas as funcionalidades/tarefas a serem desenvolvidas durante um projeto, baseadas nas necessidades do cliente/usuário.
} 
Os testes realizados com 99 usuários indicam uma melhor aceitação da proposta por parte das crianças em relação aos adolescentes participantes. Identificamos que muitos jogadores não conseguiram concluir o jogo (65.7\%), fato associado à demanda de melhoria na jogabilidade apontada por alguns usuários, sendo que tais contribuições foram identificadas e adicionadas ao backlog do projeto. Um fator bastante positivo identificado durante a avaliação do jogo desenvolvido é a percepção massiva (97\%) dos usuários de que este pode auxiliar no entendimento de conceitos relacionados a segurança na internet para crianças e adolescentes, consistindo em um forte indicador que os aspectos pedagógicos pautados na aprendizagem tangencial, habilidades e competências do mundo digital propostos pela SBC foram bem aceitos pelos jogadores.

Neste sentido, a literatura carece de trabalhos relatando a utilização de jogos educacionais customizados para o auxílio no aprendizado de conceitos relacionados a segurança na internet para crianças e adolescentes, sendo esta uma das principais contribuições de nosso estudo.

Para trabalhos futuros, pretendemos realizar ajustes na jogabilidade do Self Protect visando melhorar principalmente sua aceitação perante os adolescentes, incluir novos elementos lúdicos para torná-lo ainda mais divertido; inclusão de novas fases e desafios, proporcionando experiências imersivas sobre conceitos relacionados à segurança na internet.

\section{Referências}

Andrade, M. et al. (2012) "Coletando: Desenvolvimento de um jogo para o ensino-aprendizagem no campo da Educação Ambiental”. In: Simpósio Brasileiro de Jogos e Entretenimento Digital - SBGAMES, p.188-191.

Andrade, Pedro Henrique Mota Oliveira de; Almeida, Fábio Barros de; Bittencourt, Roberto Almeida. (2015) "Um Jogo Educacional 2D sobre Trânsito e Cidadania". In: XIV Simpósio Brasileiro de Games e Entretenimento Digital - SBGames, p.691-694.

Bloom, B.S.et al (1956). Taxonomy of educational objectives: The classification of educational goals. Handbook 1: Cognitive domain. New York: David McKay.

Brasil. (1998) "Parâmetros Curriculares Nacionais (PCNs). Temas Transversais - Ética". Ensino Fundamental - 5. a $8 .^{\mathrm{a}}$ série. Brasilia: $\mathrm{MEC} / \mathrm{SEF}$.

Bruner, Jerome Seymour (1960). Process of Education. 1.ed. New York: Vintage Books Random House.

Carvalho, Emerson A.; Reis, Thales A.; Alves, Fábio J. (2017) "Ensino de Noções Básicas de Segurança da Informação nas Escolas Brasileiras". In: Anais do XXIII Workshop de Informática na Escola (WIE 2017), p. 765-774. DOI: 10.5753/cbie.wie.2017.765.

CGI.br. (2017) "Pesquisa sobre o uso da internet por crianças e adolescentes no Brasil”. [Online. Acesso em 04 de julho de 2017].

Dias, Jéssica David et al. (2016) "Design e avaliação de um jogo educacional de anatomia e fisiologia digestória humana". In: XV Simpósio Brasileiro de Games e Entretenimento Digital - SBGames, p.288-294. 
VIII Congresso Brasileiro de Informática na Educação (CBIE 2019)

Anais do XXV Workshop de Informática na Escola (WIE 2019)

Falcão, Taciana Pontual; Barbosa, Rafael Santos (2015). “Aperta o Play!” Análise da Interação Exploratória em um Jogo Baseado em Pensamento Computacional. In: Anais do XXVI Simpósio Brasileiro de Informática na Educação (SBIE 2015), p. 419-428. DOI: 10.5753/cbie.sbie.2015.419.

Felicia, P. (2012) "Digital Games in schools: a handbook for teachers", European Schoolnet.

Krause, Katiane K.G. et al. (2017) “Jogo Sério para Auxílio no Desenvolvimento do Conceito Multiplicativo”. In: XVI Simpósio Brasileiro de Games e Entretenimento Digital - SBGames, p.269-275.

Likert, R (1932). A technique for the measurement of attitudes. Archives of psychology.

Medeiros, T. J.; Silva, T. R.; Aranha, E. H. S. (2013) "Ensino de programação utilizando jogos digitais: uma revisão sistemática da literatura”. In: Revista Novas Tecnologias na Educação RENOTE, v.11, n.3.

Novak, J. (2011) “Game development essentials: an introduction”. Ed.Cengage Learning.

Phillips, John (1972). Teoria de Piaget sobre as origens do intelecto. 1.ed. Rio de Janeiro: Universidade Aberta/ Socicultur.

Ponte, C. (2012) "Kids Online na Europa e no Brasil. Desafios para a pesquisa comparada sobre as práticas de crianças e adolescente na Internet”. Comunicação, Mídia e Consumo, 9 (25), 13 42 .

Schell, J. (2011) “A Arte de Game Design: O Livro Original”. Rio de Janeiro, Brasil: Elsevier.

Silva, Deivid Eive el al. (2015) "A tecnologia no auxílio à inserção dos temas transversais aos conteúdos das disciplinas do Ensino Fundamental: Uma experiência na Região Norte". In: Anais do IV Congresso Brasileiro de Informática na Educação (CBIE 2015), p. 703-711.

Silva, Tatyane Souza Calixto da; Melo, Jeane Cecília Bezerra; Tedesco, Patricia Cabral de Azevedo Restelli (2016). Um modelo para promover o engajamento estudantil no aprendizado de programação utilizando gamification. In: Anais dos Workshops do V Congresso Brasileiro de Informática na Educação (CBIE 2016), p. 71-80. DOI: 10.5753/cbie.wcbie.2016.71.

Silva, Thiago Reis da. (2017) “Desenvolvendo a Programação de Jogos Digitais no Ensino Médio: um Relato de Experiência Utilizando a Ferramenta Construct 2". In: Anais do VI Congresso Brasileiro de Informática na Educação (WCBIE 2017), p. 1142-1151.

Sociedade Brasileira De Computação (2017). "Referenciais de Formação em Computação: Educação Básica”. Disponível em: <http://bit.ly/2LMcevX>. Acesso em 18 jun. 2018.

Tarouco, Liane Margarida Rockenbach et al. (2004) "Jogos educacionais". Revista Novas Tecnologias na Educação, Rio Grande do Sul, v.2, n. 1.

Veras, M. (2016). “Gestão dinâmica de projetos: Life Cycle Canvas”. Rio de Janeiro: Brasport. 